\title{
Rare primary patellar resurfacing does not lead to more secondary patellar resurfacing: analysis of 70,014 primary total knee arthroplasties in the Dutch Arthroplasty Register (LROI)
}

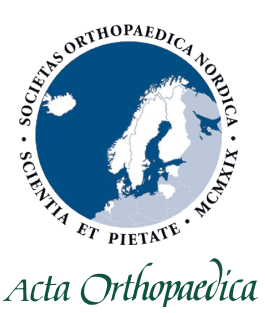

\author{
Bart J ROBBEN ${ }^{1}$, Astrid J DE VRIES ${ }^{1}$, Anneke SPEKENBRINK-SPOOREN ${ }^{2}$, \\ Rob G H H NELISSEN ${ }^{2,3}$, and Reinoud W BROUWER ${ }^{1}$
}

\author{
${ }^{1}$ Department of Orthopedic Surgery, Martini Hospital Groningen, Groningen, The Netherlands; ${ }^{2}$ Dutch Arthroplasty Register \\ (LROI), 's-Hertogenbosch, The Netherlands; ${ }^{3}$ Department of Orthopedics, Leiden University Medical Center, Leiden, The \\ Netherlands \\ Correspondence: bjrobben@gmail.com \\ Submitted 2021-09-05. Accepted 2022-01-20.
}

Background and purpose - Current literature provides no conclusive evidence in support of a patellar resurfacing vs. non-resurfacing regime. Therefore, we compared the incidence of secondary patellar resurfacing among hospitals using 3 different primary patellar resurfacing regimes in the Netherlands. Secondarily we identified patient and surgical characteristics associated with primary patellar resurfacing and secondary patella resurfacing following non-resurfaced primary total knee arthroplasty (TKA).

Patients and methods - We used data from 2014-2016 of the Dutch Arthroplasty Register. Hospitals were divided into rare (0-10\%), selective (>10\% to $90 \%)$, and usually primary patellar resurfacing $(>90 \%)$ regimes. We performed a logistic regression analysis for associated factors of primary patellar resurfacing in the selective resurfacing subgroup and for secondary patellar resurfacing in the rare resurfacing subgroup.

Results - The rate of primary resurfacing was $5.2 \%$ for the rare and $36 \%$ for the selective patellar resurfacing regimes, with similar secondary patellar resurfacing $(1.1 \%$ vs. $0.9 \%)$. Predictors for primary patellar resurfacing were being female (OR 1.3) and younger (50-59 years, OR 1.4). The PS prosthesis design had a higher OR (4.1) than the CR design. Younger age (50-59 years, OR 1.5) and PS prosthesis (OR 2.7) were significant predictors of secondary patellar resurfacing. Particular surgical systems have a higher rate of primary and secondary patellar resurfacing.

Interpretation - Low rates of secondary patellar resurfacing in hospitals with a rare resurfacing regime indicate that this regime does not lead to more secondary patellar resurfacing then selective resurfacing. In the Dutch orthopedic community primary and secondary patellar resurfacing is associated with using a posterior stabilizing design, being younger, and using particular TKA systems.
When performing a total knee arthroplasty (TKA) there is a choice as to whether to resurface the patella. The percentage of patellar resurfacing in primary TKA in the Netherlands is approximately $20 \%$ (1). This percentage is high compared with Sweden, Denmark, and Germany $(2-11 \%)$ and low compared with the UK, Australia, and USA (40-90\%) (1-4). The differences between countries seem remarkable, considering that populations and implants used are quite similar (1,5-7).

Current literature remains controversial as it provides no conclusive evidence in support of a patellar resurfacing vs. non-resurfacing regime $(\mathbf{5 , 8 , 9 )}$. A meta-analysis showed that the number of revision surgeries was lower following TKA with patellar resurfacing than without (10). However, patellar resurfacing is not without risks (11) and revision of the patellar component can be challenging due to bone loss. Costeffectiveness analysis studies provide contradictory evidence concerning routine primary patellar resurfacing (12-15). Some surgeons make the choice to resurface the patella depending on severity of the osteoarthritis on the patella, or may use a variety of criteria that might be implant- or patient-related (16). Financial reasons may also drive indications, as well as reimbursement, legal aspects, and personal preferences (17).

Maney et al. (18) investigated whether there were differences in outcomes between strategies for patellar resurfacing by dividing surgeons into 1 of 3 regimes: "usually" (>90\%), "selective' $(10-90 \%)$, or "rarely" $(<10 \%)$ resurface the patella and found that there was no difference in the revision rates. Currently there are no other studies comparing these regimes.

In this national registry study we evaluate and compare the incidence of secondary patellar resurfacing between hospitals using 3 different primary patellar resurfacing regimes in the Netherlands. Secondarily we identify patient and surgical characteristics associated with primary patellar resurfacing and secondary patellar resurfacing following non-resurfaced primary TKA. 


\section{Patients and methods}

\section{Dutch Arthroplasty Register}

The Dutch Arthroplasty Register (LROI) is a nationwide population-based registry with information on all arthroplasties performed in the Netherlands. The registry started in 2007, and $100 \%$ coverage of all Dutch hospitals was achieved in 2012. The reporting of arthroplasties in the registry increased over time, resulting in a completeness of $96 \%$ for primary TKAs in 2012 and $90 \%$ for knee revision arthroplasties in 2013 (19). The LROI contains information on patient characteristics and surgery and implant characteristics. The opt-out system is used by the LROI, requiring informed consent of patients.

\section{Data selection}

In the course of time 3 variables were added to the registry (BMI, smoking, and Charnley score) and became obligatory in 2014. Data completeness is published in the annual reports (1). As a minimum follow-up of 3 years after the primary surgery was considered clinically relevant, LROI TKA registry data until December 31, 2019 was obtained and all patients with primary osteoarthritis aged 18 years and older with a primary TKA between January 1, 2014 and January 1, 2017 were included.

\section{Patellar resurfacing subgroups}

To compare different regimes for primary patellar resurfacing hospitals were divided into 3 subgroups: rare patellar resurfacing, selective patellar resurfacing, usually patellar resurfacing. Rare patellar resurfacing was defined as primary patellar resurfacing in $0-10 \%$ of TKAs, selective patellar resurfacing in $>10 \%$ to $90 \%$ of TKAs, and usually patellar resurfacing in $>90 \%$ of TKAs (18). Hospital strategy rather than surgeon strategy was analyzed as Dutch and EU privacy legislation prevents the LROI from collecting the resurfacing rates of individual surgeons (20). Nonetheless, resurfacing rate thresholds are consistent with a previous study analyzing individual surgeon strategy (18). Uniformity in treatment protocols within hospitals is expected because of frequent and mandatory quality control of all Dutch hospitals and clinics. Every 2-5 years an independent quality committee of the Dutch Orthopedic Society reviews each group of orthopedic surgeons within a hospital. They evaluate differences in performance, adherence to national or local guidelines, and outcome of performance indicators. The committee gives compulsory advice, striving for quality improvement and uniformity within a hospital.

To reduce the number of variables we applied a second selection criterion and included only the 5 most commonly used knee prostheses in the Netherlands: Genesis II (Smith \& Nephew, Memphis, TN, USA), Nexgen (Zimmer Biomet, Warsaw, IN, USA), Vanguard (Zimmer Biomet, Warsaw, IN, USA), PFC/Sigma (DePuy Synthes, Warsaw, IN, USA), and
LCS (DePuy Synthes, Warsaw, IN, USA). These 5 surgical systems compose $84 \%$ of all knee prostheses used in the Netherlands in 2014-2016.

We selected the subgroup which performed TKA with a selective patellar resurfacing regime to analyze the associated factors for primary patellar resurfacing. The analysis of secondary patellar resurfacing was done in the rare patellar resurfacing regime, as in this group the possible influence of selection criteria for primary patellar resurfacing is less of an issue. The same selection criteria as in the subgroup for primary resurfacing (years 2014-2016 and 5 most commonly used TKA surgical systems) were used and those patients who had no primary patellar resurfacing were analyzed.

Revision surgery is defined as interchange or addition of a component. Within the LROI, revision surgery is categorized based on the components that have been changed or added. Secondary patellar resurfacing is a separate category and thus could be easily identified.

\section{Included variables}

Patient characteristics collected in the LROI were: age, sex (BMI, smoking (yes/no), ASA (I-IV), and Charnley score $(\mathrm{A}-\mathrm{C})$. All variables were made categorical and cases with extreme values (age $>105$ years and BMI $<10$ and $>70$ ) were seen as registration or classification errors and recorded as missing $(\mathrm{n}=27)$.

Surgery and implant characteristics included in this study were: type of knee prosthesis (cruciate retaining [CR] or posterior stabilized [PS]), insert mobility (fixed/rotating), surgical system (Genesis II/Nexgen/Vanguard/PFC Sigma/LCS), fixation (cemented/hybrid/uncemented), and surgical approach (medial parapatellar/lateral parapatellar/subvastus).

\section{Statistics}

Frequencies and percentages of primary and secondary patellar resurfacing were calculated for the total group and for the subgroups. To compare the proportion of secondary patellar resurfacing between the subgroups we used a Pearson chisquare test. Differences in duration of the median survival in years between subgroups was assessed using the Mann-Whitney U-test.

In the selective and rare patellar resurfacing subgroups, univariable and multivariable logistic regression analyses were used to assess the factors associated with patellar resurfacing. For the primary patellar resurfacing analysis the outcome variable was primary patellar resurfacing (yes/no) and for the multivariable analysis a stepwise Backward Likelihood Ratio model was used, because in this study no hypothesis was tested and because of the suppressor effects that are less likely to occur than in a forward selection method (21). All patient and surgery/implant characteristics were included. For the secondary patellar resurfacing analysis the outcome variable was secondary patellar resurfacing (yes/no), and because of the relative small sample of cases only those vari- 


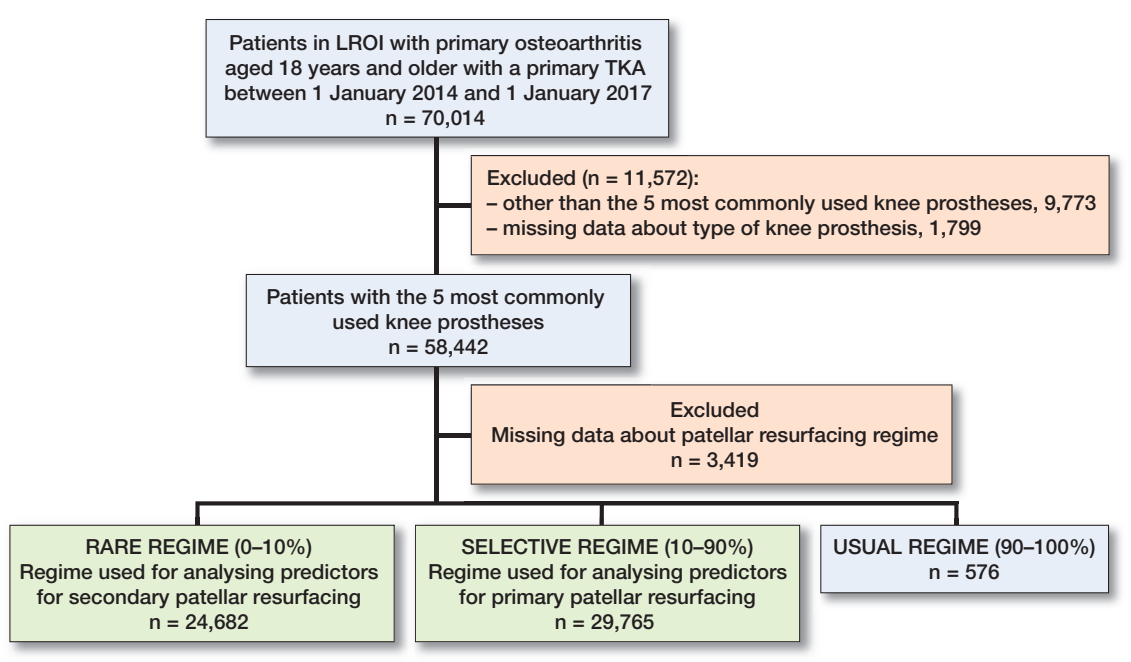

Flow chart of the numbers of patients that were excluded.

\section{Results}

In 2014-2016, 70,014 primary TKAs for osteoarthritis were registered in the Netherlands with a mean follow-up of 4.3 years (SD 1.1), 15,017 of which had primary patellar resurfacing $(21 \%)$. Patellar resurfacing rates were stable for 2014-2016 (2014 $=21 \%, 2015=22 \%, 2016=22 \%) .54,997$ patients had no primary patellar resurfacing TKA in this period, and $566(1 \%)$ of them were registered as having secondary patellar resurfacing.

Selecting the 5 most commonly used knee prostheses, 55,023 primary TKAs were available for further analysis (Flow chart). 46 hospitals used a rare primary patellar

Table 1. Numbers of TKAs and primary and secondary patellar resurfacing among the 3 primary patellar resurfacing regimes. Values are frequency (\%)

\begin{tabular}{lrrrr}
\hline Regime & TKAs & primary & $\begin{array}{c}\text { Patellar resurfacing } \\
\text { no primary }\end{array}$ & secondary \\
\hline Rare & 24,682 & $1,277(5.2)$ & 23,405 & $255(1.1)$ \\
Selective & 29,765 & $10,740(36)$ & 19,025 & $180(0.9)$ \\
Usually & 576 & $568(99)$ & 8 & $0(-)$ \\
\hline
\end{tabular}

Rare $0-10 \%$; Selective $>10 \%$ to $90 \%$; Usually $>90 \%$

ables were included in the multivariable analysis that had a significant association in the univariable analysis. Frequencies and percentages were used to present the data of all variables included in the logistic regression model. Categories with fewer than 10 patients are considered not reliable and are not presented (displayed in the results as "not available"). IBM SPSS version 20.0 was used for the statistical analysis (IBM Corp, Armonk, NY, USA) and a p-value below 0.05 was considered statistically significant. The confidence interval (CI) was $95 \%$.

\section{Ethics, funding, data sharing and potential conflicts of interest}

In this study the data from the LROI is used. Due to this there is no approval by a local ethics committee. Projects submitted by the LROI are evaluated by its Scientific Advisory Board. The Scientific Advisory Board of the LROI gave positive advice concerning the project (approval on February 9, 2020). The authors did not receive any funding or have any conflict of interest regarding this article. Sharing data from the Dutch Arthroplasty Register as used for this study is not possible. The General terms and conditions of data usage from the Dutch Arthroplasty Register contains a statement that LROI data cannot be shared. resurfacing regime (range $0.1-9.9 \%$, median $2.6 \%$ ), 37 hospitals used a selective (range 10-76\%, median 26\%), and 4 hospitals used a usual (range: 90-97\%, median 97\%) patellar resurfacing regime.

In the group rare patellar resurfacing, $255(1.1 \%)$ patients had a secondary patellar resurfacing, compared with 180 $(0.9 \%)$ patients in the group selective patellar resurfacing (Table 1). The group usually patellar resurfacing is small (4.6\% of the hospitals and 576 [1\%] TKAs in total) of which only 8 had no primary patellar resurfacing. No statistically significant differences were found in the proportions of secondary patellar resurfacing between the rare and selective primary resurfacing groups $(\mathrm{p}=0.2)$. Secondary patellar resurfacing was performed with a median interval of 1.7 years (range $0-6$ ) in the rare patellar resurfacing group and with a median interval of 1.6 years (range $0-5$ ) in the selective patellar resurfacing group, which was not statistically significant $(p=0.8)$. The main reasons for secondary patellar resurfacing were anterior knee pain (92\%) and patellar dislocation (6\%) (multiple reasons could be indicated by the surgeon).

We used the subgroup that performed TKA with a selective patellar resurfacing regime to analyze the factors associated with primary patellar resurfacing; for the associated factors with secondary patellar resurfacing we used the rare regime subgroup (Flow chart, Table 2).

\section{Patient characteristics for primary patellar resurfacing}

Women had increased odds (OR 1.3, CI 1.2-1.3) of having primary patellar resurfacing compared with men. Other significant predictors were younger age (50-59 years, OR 1.5 , CI $1.2-1.8)$ compared with the reference category ( $70-79$ years) and a higher Charnley score (osteoarthritis of multiple joints Charnley B1 [OR 1.1, CI 1.1-1.2], B2 [OR 1.3, CI 1.2-1.4]) compared with Charnley A (Table 3). Based on the multivariable analysis, patellar resurfacing was comparable for BMI categories, smoking, and ASA classification. 
Table 2. Baseline characteristics of primary and secondary patellar resurfacing in primary total knee arthroplasties for osteoarthritis in the Netherlands for 2014-2016. Values are frequency (\%)

\begin{tabular}{|c|c|c|c|c|}
\hline \multirow[b]{2}{*}{ Factor } & \multicolumn{2}{|c|}{$\begin{array}{l}\text { Primary patellar } \\
\text { resurfacing }{ }^{\text {a }}\end{array}$} & \multicolumn{2}{|c|}{$\begin{array}{l}\text { Secondary patellar } \\
\text { resurfacing } \mathbf{b}\end{array}$} \\
\hline & $\begin{array}{c}\text { Yes } \\
10,740(36)\end{array}$ & $\begin{array}{c}\text { No } \\
19,025(64)\end{array}$ & $\begin{array}{c}\text { Yes } \\
255(1.1)\end{array}$ & $\begin{array}{c}\text { No } \\
23,150(90)\end{array}$ \\
\hline \multicolumn{5}{|c|}{ Patient characteristics } \\
\hline \multicolumn{5}{|c|}{ Sex } \\
\hline Male & $3,551(33)$ & $7,040(37)$ & $77(30)$ & $8,321(36)$ \\
\hline Female & 7,179 (67) & $11,958(63)$ & $178(70)$ & $14,809(64)$ \\
\hline Missing & $10(0.1)$ & $27(0.1)$ & $0(0.0)$ & $20(0.1)$ \\
\hline \multicolumn{5}{|l|}{ Age } \\
\hline $18-49$ & $215(2.0)$ & $317(1.7)$ & $8(3.1)$ & $387(1.7)$ \\
\hline $50-59$ & $1,611(15)$ & 2,407 (13) & $52(20)$ & 3,185 (14) \\
\hline $60-69$ & 3,930 (37) & $6,833(36)$ & $104(41)$ & $8,214(36)$ \\
\hline 70-79 & $3,732(35)$ & 7,096 (37) & $80(31)$ & $8,420(36)$ \\
\hline $80-100$ & $1,250(12)$ & 2,371 (13) & 11 (4.3) & 2,939 (13) \\
\hline Missing & $2(0.0)$ & $1(0.0)$ & $0(0)$ & $5(0.0)$ \\
\hline \multicolumn{5}{|l|}{ BMI } \\
\hline$<18.5$ & $19(0.2)$ & $25(0.1)$ & $2(0.8)$ & $30(0.1)$ \\
\hline $20-25$ & $1,588(15)$ & 2,927 (15) & $33(13)$ & $3,642(16)$ \\
\hline $25-30$ & 4,125 (38) & 7,327 (39) & $81(32)$ & $9,283(40)$ \\
\hline $30-40$ & $4,289(40)$ & $7,203(38)$ & $110(43)$ & $8,557(37)$ \\
\hline$>40$ & $485(4.5)$ & $760(4.0)$ & 7 (2.7) & 835 (3.6) \\
\hline Missing & $234(2.2)$ & $783(4.1)$ & $22(8.6)$ & $803(3.5)$ \\
\hline \multicolumn{5}{|l|}{ Smoking } \\
\hline No & $9112(85)$ & $15180(80)$ & $183(72)$ & $19046(82)$ \\
\hline Yes & $1033(9.6)$ & $1488(7.8)$ & $35(14)$ & 2039 (8.8) \\
\hline Missing & $595(5.5)$ & $2357(12)$ & $37(15)$ & 2065 (8.9) \\
\hline \multicolumn{5}{|l|}{ ASA } \\
\hline 1 & $1,380(13)$ & $2,453(13)$ & $40(16)$ & $3,367(15)$ \\
\hline II & $7,715(72)$ & $13,463(71)$ & $167(66)$ & $15,642(68)$ \\
\hline III-IV & $1,620(15)$ & $3,070(16)$ & $46(18)$ & $4,106(18)$ \\
\hline Missing & $25(0.2)$ & $39(0.2)$ & $2(0.8)$ & $35(0.2)$ \\
\hline \multicolumn{5}{|l|}{ Charnley } \\
\hline A & 3,949 (37) & $7,723(41)$ & $132(52)$ & $10,008(43)$ \\
\hline B1 & 3,907 (36) & $6,387(34)$ & 78 (31) & 3 (33) \\
\hline B2 & $2,294(21)$ & 3,530 (19) & $26(10)$ & $4,439(19)$ \\
\hline C & $300(2.8)$ & $467(2.5)$ & $4(1.6)$ & $614(2.7)$ \\
\hline Missing & $290(2.7)$ & $918(4.8)$ & $15(5.9)$ & $556(2.4)$ \\
\hline \multicolumn{5}{|c|}{ Surgical/Implant characteristics } \\
\hline \multicolumn{5}{|c|}{ Approach } \\
\hline \multicolumn{5}{|c|}{ Parapatellar } \\
\hline medial & $10,163(95)$ & $18,040(95)$ & $245(96)$ & $22,263(96)$ \\
\hline lateral & $70(0.7)$ & $133(0.7)$ & $2(0.8)$ & $286(1.2)$ \\
\hline Vastus & $473(4.4)$ & $806(4.2)$ & $6(2.4)$ & $544(2.3)$ \\
\hline Missing & $34(0.3)$ & $46(0.2)$ & $2(0.8)$ & $57(0.2)$ \\
\hline \multicolumn{5}{|c|}{ Type of knee prosthesis } \\
\hline CR & $2,517(23)$ & $8,410(44)$ & $89(35)$ & $12,677(55)$ \\
\hline PS & $8,176(76)$ & $10,579(56)$ & $166(65)$ & $10,429(45)$ \\
\hline Missing & $47(0.4)$ & $36(0.2)$ & $0(0.0)$ & $44(0.2)$ \\
\hline \multicolumn{5}{|c|}{ Insert mobility } \\
\hline Fixed & $10,401(97)$ & $17,945(94)$ & $214(84)$ & $17,148(74)$ \\
\hline Rotating & $41(0.4)$ & $957(5.0)$ & $40(16)$ & $5,858(25)$ \\
\hline Missing & $298(2.8)$ & $123(0.6)$ & $1(0.4)$ & $144(0.6)$ \\
\hline \multicolumn{5}{|c|}{ Fixation } \\
\hline Cemented & $10,556(98)$ & $17,986(95)$ & $222(87)$ & $20,128(87)$ \\
\hline Uncemente & ed $22(0.2)$ & $40(0.2)$ & 7 (2.7) & $1,547(6.7)$ \\
\hline Hybrid & $144(1.3)$ & $958(5.0)$ & $26(10.2)$ & ) $1,448(6.3)$ \\
\hline Missing & $18(0.2)$ & $41(0.2)$ & $0(0.0)$ & $27(0.1)$ \\
\hline Surgical syste & em & & & \\
\hline Genesis II & $2,456(23)$ & 6,089 (32) & $70(28)$ & $5,509(24)$ \\
\hline Nexgen & 2,062 (19) & $5,344(28)$ & $42(17)$ & $5,118(22)$ \\
\hline Vanguard & $3,233(30)$ & 4,195 (22) & $53(21)$ & $4,233(18)$ \\
\hline PFC/Sigma & a $2,983(28)$ & 2,452 (13) & $53(21)$ & $2,544(11)$ \\
\hline LCS & $6(0.1)$ & $945(5.0)$ & 37 (15) & $5,746(25)$ \\
\hline
\end{tabular}

a Analysis of the group with a selective patellar resurfacing regime.

${ }^{b}$ Analysis of the group with a rare patellar resurfacing regime.
Table 3. Univariable and multivariable logistic regression analysis for primary patellar resurfacing

\begin{tabular}{|c|c|c|}
\hline \multirow[b]{3}{*}{ Factor } & \multicolumn{2}{|c|}{ Primary patellar resurfacing } \\
\hline & Univariable & Multivariable \\
\hline & OR $(95 \% \mathrm{Cl})$ & OR $(95 \% \mathrm{Cl})$ \\
\hline \multicolumn{3}{|l|}{ Patient characteristics } \\
\hline Female sex (ref. male) & $1.2(1.1-1.3)^{b}$ & $1.3(1.2-1.3)^{b}$ \\
\hline \multicolumn{3}{|l|}{ Age (ref. 70-79) } \\
\hline $18-49$ & $1.3(1.1-1.6)^{a}$ & $1.5(1.2-1.8)^{\mathrm{a}}$ \\
\hline $50-59$ & $1.3(1.2-1.4)^{\mathbf{b}}$ & $1.4(1.3-1.5)^{b}$ \\
\hline $60-69$ & $1.1(1.0-1.2)^{b}$ & $1.1(1.1-1.2)^{b}$ \\
\hline 80-100 & $1.0(0.9-1.1)$ & $0.95(0.9-1.1)$ \\
\hline \multicolumn{3}{|l|}{ BMI (ref. 20-25) } \\
\hline$<18.5$ & $1.3 \quad(0.7-2.4)$ & $1.8(0.9-3.5)$ \\
\hline $25-30$ & $1.0(1.0-1.1)$ & $1.1(1.0-1.2)$ \\
\hline $30-40$ & $1.1(1.0-1.2)^{a}$ & $1.1(1.0-1.2)$ \\
\hline$>40$ & $1.2(1.1-1.4)^{a}$ & $1.2(1.0-1.4)$ \\
\hline Smoking (ref. no) & $1.2(1.1-1.3)^{b}$ & $1.1 \quad(1.0-1.2)$ \\
\hline \multicolumn{3}{|l|}{ ASA (ref. ASA I) } \\
\hline ASA II & $1.0(1.0-1.1)$ & $1.0(1.0-1.1)$ \\
\hline ASA III-IV & $0.95(0.9-1.0)$ & $0.93(0.8-1.0)$ \\
\hline \multicolumn{3}{|l|}{ Charnley (ref. A) } \\
\hline B1 & $1.2(1.2-1.3)^{b}$ & $1.1(1.1-1.2)^{b}$ \\
\hline B2 & $1.3(1.2-1.4)^{b}$ & $1.3(1.2-1.4)^{b}$ \\
\hline $\mathrm{C}$ & $1.2(1.1-1.4)^{b}$ & $1.2(1.0-1.4)$ \\
\hline \multicolumn{3}{|c|}{ Surgical/implant characteristics } \\
\hline \multicolumn{3}{|c|}{ Approach (ref. medial parapatellar) } \\
\hline Lateral parapatellar & $0.94(0.7-1.3)$ & $1.0(0.7-1.4)$ \\
\hline Subvastus & $1.1(1.0-1.2)$ & $0.79(0.7-0.9)$ a \\
\hline \multicolumn{3}{|c|}{ Type of knee prosthesis (ref. CR) } \\
\hline PS & $2.7(2.5-2.8)^{b}$ & $4.1(3.8-4.4)^{b}$ \\
\hline \multicolumn{3}{|l|}{ Insert mobility (ref. fixed) } \\
\hline Rotating & $0.07(0.05-0.1)^{b}$ & $1.5 \quad(0.6-3.9)$ \\
\hline \multicolumn{3}{|l|}{ Fixation (ref. cemented) } \\
\hline Uncemented & $0.94(0.6-1.6)$ & $0.87(0.5-1.6)$ \\
\hline Hybrid & $0.26(0.2-0.3)^{b}$ & $0.52(0.4-0.6)^{b}$ \\
\hline \multicolumn{3}{|c|}{ Surgical system (ref. Genesis II) } \\
\hline Nexgen & $0.96(0.9-1.0)$ & $0.76(0.7-0.8)^{b}$ \\
\hline Vanguard & $1.9(1.8-2.0)^{b}$ & $3.1(2.8-3.3)^{b}$ \\
\hline PFC /Sigma & $3.0(2.8-3.2)^{b}$ & $4.2(3.9-4.6)^{b}$ \\
\hline LCS & $0.02(0.01-0.04)^{b}$ & $0.04(0.01-0.1)^{b}$ \\
\hline
\end{tabular}

a $p<0.05$

b $p<0.001$.

\section{Surgical/implant characteristics for primary patellar resurfacing}

Based on the multivariable analysis, the PS knee prosthesis design was a significant predictor for primary patellar resurfacing (OR 4.1, 95\% CI 3.82-4.40, $\mathrm{p}<0.001)$ compared with a CR design (Table 3).

Compared with the Genesis II, LCS (OR 0.04, CI 0.0-0.1) and Nexgen (OR 0.76, CI 0.7-0.8), both had significantly lower odds for primary patellar resurfacing. By contrast, $\mathrm{PFC} /$ Sigma (OR 4.2, CI 3.9-4.6) and Vanguard (OR 3.1, CI 2.93.3) had increased ORs. Primary TKA with a hybrid fixation had significantly lower odds (OR 0.52, CI 0.4-0.6) of resulting in patellar resurfacing compared with a cemented fixation. Compared with a medial parapatellar approach, the odds of resulting in patellar resurfacing for patients with a subvastus approach were lower (OR 0.79, CI 0.7-0.9). 
Table 4. Univariable and multivariable logistic regression analysis for secondary patellar resurfacing

\begin{tabular}{|c|c|c|}
\hline \multirow[b]{3}{*}{ Factor } & \multicolumn{2}{|c|}{ Secondary patellar resurfacing } \\
\hline & Univariable & Multivariable \\
\hline & OR $(95 \% \mathrm{Cl})$ & OR $(95 \% \mathrm{Cl})$ \\
\hline \multicolumn{3}{|l|}{ Patient characteristics } \\
\hline Female sex (ref. male) & $1.3(0.99-1.7)$ & \\
\hline \multicolumn{3}{|l|}{ Age (ref. 70-79) } \\
\hline $18-49$ & NA & NA \\
\hline $50-59$ & $1.7(1.2-2.4)^{a}$ & $1.5(1.0-2.3)^{a}$ \\
\hline $60-69$ & $1.3(0.99-1.8)$ & $1.2(0.8-1.7)$ \\
\hline $80-100$ & $0.39(0.2-0.7)^{a}$ & $0.35(0.2-0.7)$ a \\
\hline \multicolumn{3}{|l|}{ BMI (ref. 20-25) } \\
\hline$<18.5$ & NA & \\
\hline $25-30$ & $0.96(0.6-1.5)$ & \\
\hline $30-40$ & $1.4(0.96-2.1)$ & \\
\hline$>40$ & NA & \\
\hline Smoking (ref. no) & $1.8(1.2-2.6)^{a}$ & $1.6(1.1-2.3)^{a}$ \\
\hline \multicolumn{3}{|l|}{ ASA (ref. ASA I) } \\
\hline ASA II & $0.90(0.6-1.3)$ & \\
\hline ASA III-IV & $0.94(0.6-1.4)$ & \\
\hline \multicolumn{3}{|l|}{ Charnley (ref. A) } \\
\hline B1 & $0.79(0.6-1.0)$ & $0.78(0.6-1.1)$ \\
\hline B2 & $0.44(0.3-0.7)^{b}$ & $0.54(0.4-0.8)^{a}$ \\
\hline C & NA & NA \\
\hline \multicolumn{3}{|c|}{ Surgical/implant characteristics } \\
\hline \multicolumn{3}{|c|}{ Approach (ref. medial parapatellar } \\
\hline Lateral parapatellar & NA & \\
\hline Subvastus & NA & \\
\hline \multicolumn{3}{|l|}{ Type of knee prosthesis } \\
\hline PS & $2.3(1.8-2.9)^{a}$ & $2.7(1.9-4.0)^{b}$ \\
\hline \multicolumn{3}{|l|}{ Insert mobility (ref. fixed) } \\
\hline Rotating & $0.55(0.39-0.77)^{b}$ & $1.3 \quad(0.19-8.4)$ \\
\hline \multicolumn{3}{|l|}{ Fixation (ref. cemented) } \\
\hline Uncemented & NA & NA \\
\hline Hybrid & $1.6(1.1-2.5)^{a}$ & $1.5(0.95-2.5)$ \\
\hline \multicolumn{3}{|c|}{ Surgical system (ref. Genesis II) } \\
\hline Nexgen & $0.65(0.44-0.95)^{a}$ & $0.72(0.48-1.1)$ \\
\hline Vanguard & $0.99(0.69-1.4)$ & $1.4 \quad(0.92-2.2)$ \\
\hline PFC/Sigma & $1.6(1.1-2.5)^{a}$ & $2.1(1.3-3.4)^{a}$ \\
\hline LCS & $0.51(0.34-0.76)^{a}$ & $1.2(0.16-8.2)$ \\
\hline $\begin{array}{l}N A=\text { Not available, catego } \\
\text { a } p<0.05 ; \\
\text { b } p<0.001 .\end{array}$ & $\mathrm{n}<10$. & \\
\hline
\end{tabular}

\section{Patient characteristics for secondary patellar resur- facing}

A significant predictor for secondary patellar resurfacing was age 50-59 years (OR 1.5, CI 1.0-2.3), and 80-100 years (OR 0.35 , CI $0.17-0.73$ ) compared with the reference category (70-79 years). Patients with secondary patellar resurfacing had a mean age of 66 years (SD 8.6) compared with 69 years (SD 9.0) of patients without secondary patellar resurfacing. Smoking was found to be associated with secondary patellar resurfacing (OR 1.6, CI 1.1-2.3). Patients with the Charnley musculoskeletal comorbidity score B2 (i.e., more lower extremity involvement) had significantly lower odds (OR 0.54 , CI $0.35-0.83$ ) of resulting in secondary patellar resurfacing compared with Charnley A (Table 4). Patients with secondary patellar resurfacing had an average Charnley score of
1.6 (SD 0.75); for patients without secondary patellar resurfacing the average Charnley score was 1.8 (SD 0.84) (Charnley $\mathrm{A}=1, \mathrm{~B} 1=2, \mathrm{~B} 2=3, \mathrm{C}=4$ ).

\section{Surgical/implant characteristics for secondary patel- lar resurfacing}

The PS knee prosthesis design was a significant predictor (OR 2.7, CI 1.9-4.0) of secondary patellar resurfacing compared with a CR design (Table 4). The PFC/Sigma had an increased OR compared with the Genesis II prosthesis (OR 2.1, CI 1.33.4) (Table 4).

\section{Discussion}

The aim of this national registry study was to evaluate and compare the incidence of secondary patellar resurfacing between hospitals using three different primary patellar resurfacing regimes in the Netherlands. Our results show that secondary patellar resurfacing rates were similar in hospitals that had a rare primary patellar resurfacing compared with a selective regime. The secondary research question was to statistically identify patient and surgical characteristics associated with primary patellar resurfacing and secondary patellar resurfacing following non-resurfaced primary TKA. Significant predictors for primary as well as secondary patellar resurfacing were younger age, the PS prosthesis design, and surgical systems.

This study shows that the overall percentage of secondary patellar resurfacing in the Netherlands is only $1.0 \%$, and that in hospitals with a rare primary patellar resurfacing regime the secondary patellar resurfacing rate is only $1.1 \%$. The metaanalysis by Teel et al. (21) shows a much higher percentage of secondary patellar resurfacing, with $4.6 \%$ patellar surgery for AKP and $1.2 \%$ for reasons other than AKP. The percentage of secondary patellar resurfacing in the subgroup with selective resurfacing clinics in this study was only $0.9 \%$, which is similar to the results of the Norwegian Arthroplasty Register (0.82\%) (3). Recent data from the Swedish and Australian arthroplasty registries show that the percentage of secondary patellar resurfacing in Sweden is $0.7 \%$ and in Australia 1.7\%. This is remarkable, as the percentage of primary patellar resurfacing in Sweden is $2.6 \%$ and that of Australia is $73 \%(6,7)$. Thus, less primary patellar resurfacing does not necessarily lead to a higher percentage of secondary patellar resurfacing. Our results are in agreement with these previous findings, since based on our data a rare patellar resurfacing regime in the Netherlands does not lead to more secondary patellar resurfacing than the selective resurfacing regime. This might suggest that one could be conservative regarding primary patellar resurfacing, even though it is important to consider that the complications and clinical outcomes are not taken into account in this study and might give a different perspective. Only a small number of hospitals perform a "usually primary patellar resurfacing" regime. 
This means that there is only a small group in the Netherlands that believes in routine patellar resurfacing.

Evidence on patient, surgical, and implant characteristics influencing outcome of primary patellar resurfacing is still limited (22). A retrospective study by Maradit-Kremers et al. (16) showed that surgeons regard normal cartilage, younger age, and a thin patella as reasons for not resurfacing the patella. By contrast, we found an association with both primary and secondary patellar resurfacing in younger patients (50-59 years). Activity level and expectations may explain a higher risk of primary patellar resurfacing for these younger patients. Some implant designs (as well as some surgical systems) were likewise associated with patellar resurfacing. As for the overall concept of a PS design, it causes more posterior tibial translation due to the more anterior center of rotation, which potentially may lead to more anterior knee pain (23). The latter will be an indication for surgeons to place a patellar component preemptively.

The LCS knee system, a mobile bearing design, was the system least associated with patellar resurfacing of all studied systems, while both the Vanguard and the PFC/Sigma had a clear positive association with primary patellar resurfacing. Surgeon experience and training with a particular surgical system can influence the decision for selective patellar resurfacing, although several studies have shown that prosthetic design has limited influence on anterior knee pain and outcome after the procedure (24). Some surgical systems are marketed as patella-friendly femoral designs, which suggests less anterior knee pain (AKP) and no need for patellar resurfacing. However, Atzori et al. (25) did not show any difference in AKP between classical and patella-friendly femoral components. Our study nonetheless shows that LCS was the least associated with primary patellar resurfacing and also had a low association with secondary patellar resurfacing. This may suggest that this knee system is patella friendly. Our study does not include clinical outcomes so difference in resurfacing rate could also be due to other factors such as marketing claims or surgical training.

Several studies show an increased risk of secondary patellar resurfacing with the PS prosthesis design, in agreement with our findings $(18,26)$. Complaints like patellar clunk and crepitus $(12,13,27)$ may be a reason for increased incidence of primary and secondary patellar resurfacing with a PS prosthesis. Our results show a limited effect of surgical system on secondary patellar resurfacing. Only the PFC/Sigma system had a statistically significantly higher association with secondary patellar resurfacing compared with the Genesis II system. The metaanalysis of Pavlou et al. (24) showed no difference in secondary patellar resurfacing between patella-friendly and non-patellafriendly prostheses, suggesting that the influence of surgical system on secondary patellar resurfacing is limited. Influence of younger age and PS prosthesis design on less secondary patellar resurfacing is also shown by Maney et al. (28). Our results are similar to what previous studies have found and add to the growing body of literature around predictors of secondary patellar resurfacing. This may aid in clinical practice as surgeons may have a lower threshold for primary patellar resurfacing in younger patients, especially when using PS designs.

A major strength of this register study is that the LROI is a registry with more than $95 \%$ completeness. Furthermore, this registry is a suitable database to compare different resurfacing strategies, considering that the resurfacing rates vary statistically between hospitals. Our study has some limitations. First, the numbers in the usually resurfacing group are small and because it was so underpowered there were no secondary patellar resurfacing cases in this group. Therefore this group could not be compared with the rare or selective patellar resurfacing regimes. Second, the registry does not include clinical symptoms like anterior knee pain, patellar osteoarthritis, or other specific details of the patella. The presence of osteoarthritis of the patella may influence the decision to perform primary patellar resurfacing, although some authors find that this presence does not influence the outcome of the resurfacing $(\mathbf{8 , 2 9})$. Third, the registry registers the rate only of patellar resurfacing of hospitals, not of individual surgeons. Despite the fact that - because of frequent and mandatory quality control-uniformity in treatment protocols within hospitals is expected, slight variation between individual surgeons within a hospital may be present and may have caused a slight bias. Furthermore, our study has a maximum follow-up of 6 years, which is relatively short for an arthroplasty study. Progression of patellofemoral osteoarthritis or wear and loosening of a patellar component can occur after 6 years. However, similarly to other literature the median duration between primary TKA and secondary patellar resurfacing is 1.6 and 1.7 years, therefore the majority of revision surgeries are probably included in the analysis $(3,18,30)$.

In conclusion, the incidence of primary and secondary patellar resurfacing in the Netherlands is $21 \%$ and $1.0 \%$, respectively. The rate of secondary patellar resurfacing is similar between rare $(1.1 \%)$ and selective $(0.9 \%)$ primary patellar resurfacing regimes, while the rates of primary patellar resurfacing in these groups were 5.2 and $36 \%$ respectively. These low rates of secondary patellar resurfacing in hospitals with a rare resurfacing regime indicate that a rare patellar resurfacing regime does not lead to more secondary patellar resurfacing. Primary and secondary patellar resurfacing is mainly associated with using a posterior stabilizing design, being younger, and using particular TKA systems.

BR: design and interpretation of the work and analysis of the data. AV: design of the work and analysis of the data and revising. AS: design of the work and revising. RN: interpretation of work and revising. RB: design and interpretation of the work and revising.

Acta thanks Alistair Maney and Nanne Kort for help with peer review of this study. 
1. Dutch Arthroplasty Register (LROI). Previous annual online report of LROI. https://www.lroi-report.nl/previous-reports/ [Accessed 28 January 2021].

2. Aunan E, Næss G, Clarke-Jenssen J, Sandvik L, Kibsgård T J. Patellar resurfacing in total knee arthroplasty: functional outcome differs with different outcome scores. A randomized, double-blind study of 129 knees with 3 years of follow-up. Acta Orthop 2016; 87(2): 158-64.

3. Leta T H, Lygre S H L, Skredderstuen A, Hallan G, Jan-Erik Gjertsen J-E, Rokne B, et al. Secondary patella resurfacing in painful non-resurfaced total knee arthroplasties: a study of survival and clinical outcome from the Norwegian Arthroplasty Register (1994-2011). Int Orthop 2016; 40(4): 715-22.

4. Fraser J F, Spangehl M J. International rates of patellar resurfacing in primary total knee arthroplasty, 2004-2014. J Arthroplasty 2017; 32(1): 83-6.

5. Ali A, Lindstrand A, Nilsdotter A, Sundberg M. Similar patientreported outcomes and performance after total knee arthroplasty with or without patellar resurfacing. Acta Orthop 2016; 87(3): 274-9. doi: 10.3109/17453674.2016.1170548

6. Swedish Knee Arthroplasty Register. Annual Report 2020, http:// myknee.se/en/; 2020 [Accessed 7 June 2021].

7. Australian Orthopaedic Association National Joint Replacement Registry. Annual Report 2020, https://aoanjrr.sahmri.com/annualreports-2020; 2020 [Accessed 7 June 2021].

8. Van Raaij T M, Van der Meij E, de Vries A J, Van Raay J J A M. Patellar resurfacing does not improve clinical outcome in patients with symptomatic tricompartmental knee osteoarthritis: an RCT study of 40 patients receiving primary cruciate retaining total knee arthroplasty. J Knee Surg 2020; May 20. doi: 10.1055/s-0040-1710369. Online ahead of print.

9. Schindler $\mathbf{O} \mathbf{S}$. The controversy of patellar resurfacing in total knee arthroplasty: ibisne in medio tutissimus? Knee Surg Sports Traumatol Arthrosc 2012; 20(7): 1227-44.

10. Spencer S J, Young D, Blyth $\mathbf{M} \mathbf{J}$ G. Secondary resurfacing of the patella in total knee arthroplasty. Knee 2010; 17(3): 187-90

11. Bischoff M J F J, van Raaij T M, Reininga I H F, van Raay J J A M. Patellar resurfacing in posterior cruciate ligament retaining total knee arthroplasty (PATRES): design of a randomized controlled clinical trial. BMC Musculoskelet Disord 2014; 15(1): 1.

12. Ogawa H, Matsumoto K, Akiyama $\mathbf{H}$. Effect of patellar resurfacing on patellofemoral crepitus in posterior-stabilized total knee arthroplasty . J Arthroplasty 2016; 31(8): 1792-6.

13. Hwang B-H, Nam C-H, Jung K-A, Ong A, Lee S-C. Is further treatment necessary for patellar crepitus after total knee arthroplasty? Clin Orthop Relat Res 2013; 471(2): 606-12.

14. Zmistowski B J, Fillingham Y A, Salmons H I, Ward DT, Good R P, Lonner J H. Routine patellar resurfacing during total knee arthroplasty is not cost-effective in patients without patellar arthritis. J Arthroplasty 2019; 34(9): 1963-8.

15. Weeks C A, Marsh J D, MacDonald S J , Graves S, Vasarhelyi E M. Patellar resurfacing in total knee arthroplasty: a cost-effectiveness analysis. J Arthroplasty 2018; 33(11): 3412-15.

16. Maradit-Kremers H, Haque O J, Kremers W K, Berry D J, Lewallen D G, Trousdale $\mathbf{R} \mathbf{T}$, et al. Is selectively not resurfacing the patella an acceptable practice in primary total knee arthroplasty? J Arthroplasty 2017; 32(4): 1143-7.
17. Murray D W, MacLennan G S, Breeman S, Dakin H A, Johnston L, Campbell M K, et al. A randomised controlled trial of the clinical effectiveness and cost-effectiveness of different knee prostheses: the Knee Arthroplasty Trial (KAT). Health Technol Assess 2014; 18(19): 1-235, vii-viii.

18. Maney A J, Koh C K, Frampton C M, Young S W. Usually, selectively, or rarely resurfacing the patella during primary total knee arthroplasty. J Bone Joint Surg 2019; 101: 412-20.

19. Van Steenbergen L N, Denissen G A W, Spooren A, van Rooden S M, van Oosterhout F J, Morrenhof J W, et al. More than 95\% completeness of reported procedures in the population based Dutch Arthroplasty Register: external validation of 311890 procedures. Acta Orthop 2015; 86(4): 498-505.

20. Euro lex [internet]. Strasbourg, France: European Parliament, Council of the European Union [cited 2022 Jan 14]. Regulation (EU) 2016/679 of the European Parliament and of the Council of 27 April 2016 on the protection of natural persons with regard to the processing of personal data and on the free movement of such data, and repealing Directive 95/46/ EC (General Data Protection Regulation). Available from: https://eur-lex. europa.eu/legal-content/EN/TXT/?uri=celex\%3A32016R0679

21. Field A. Discovering statistics using SPSS. 2nd ed. London: Sage; 2005.

22. Teel A J, Esposito J G, Lanting B A, Howard J L, Schemitsch E H. Patellar resurfacing in primary total knee arthroplasty: a meta-analysis of randomized controlled trials. J Arthroplasty 2019; 34(12): 3124-32.

23. van Jonbergen H-P W, Reuver J M, Mutsaerts E L, Poolman R W. Determinants of anterior knee pain following total knee replacement: a systematic review. Knee Surg. Sports Traumatol Arthrosc 2014; 22: 47899.

24. Pavlou G, Meyer C, Leonidou A, As-Sultany M, West R, Tsiridis E. Patellar resurfacing in total knee arthroplasty: does design matter? A meta-analysis of 7075 cases. J Bone Joint Surg Am 2011; 93(14): 1301-9.

25. Atzori F, Sabatini L, Deledda $\mathbf{D}$, Schirò $\mathbf{M}$, Lo Baido $\mathbf{R}$, Massè $\mathbf{A}$. Evaluation of anterior knee pain in a PS total knee arthroplasty: the role of patella-friendly femoral component and patellar size. Musculoskelet Surg 2015; 99(1): 75-83.

26. Coory J A, Tan K G, Whitehouse S L, Hatton A, Graves S E, Crawford R W. The Outcome of total knee arthroplasty with and without patellar resurfacing up to 17 years: a report from the Australian Orthopaedic Association National Joint Replacement Registry. J Arthroplasty 2020; 35(1): 132-8.

27. Thiengwittayaporn S, Srungboonmee K, Chiamtrakool B. Resurfacing in a posterior-stabilized total knee arthroplasty reduces patellar crepitus complication: a randomized, controlled trial. J Arthroplasty 2019; 34(9): 1969-74.

28. Maney A J, Frampton C M, Young S W. Age and prosthetic design as risk factors for secondary patella resurfacing. J Arthroplasty 2020; 35(6): 1563-8.

29. Barrack R L, Bertot A J, Wolfe M W, Waldman D A, Milicic M, Myers L. Patellar resurfacing in total knee arthroplasty: a prospective, randomized, double-blind study with five to seven years of follow-up. J Bone Joint Surg Am 2001; 83(9): 1376-81.

30. Breeman S, Campbell M, Dakin H, Fiddian N, Fitzpatrick R, Grant A, et al. Patellar resurfacing in total knee replacement: five-year clinical and economic results of a large randomized controlled trial. J Bone Joint Surg Am 2011; 93(16): 1473-81. 